\title{
ON A GENERALIZATION OF HIRZEBRUCH'S THEOREM TO BOTT TOWERS
}

\author{
Jin Hong KIM
}

\begin{abstract}
The primary aim of this paper is to generalize a theorem of Hirzebruch for the complex 2-dimensional Bott manifolds, usually called Hirzebruch surfaces, to more general Bott towers of height $n$. To do so, we first show that all complex vector bundles of rank 2 over a Bott manifold are classified by their total Chern classes. As a consequence, in this paper we show that two Bott manifolds $B_{n}\left(\alpha_{1}, \ldots, \alpha_{n-1}, \alpha_{n}\right)$ and $B_{n}\left(\alpha_{1}, \ldots, \alpha_{n-1}, \alpha_{n}^{\prime}\right)$ are isomorphic to each other, as Bott towers if and only if both $\alpha_{n} \equiv \alpha_{n}^{\prime} \bmod 2$ and $\alpha_{n}^{2}=\left(\alpha_{n}^{\prime}\right)^{2}$ hold in the cohomology ring of $B_{n-1}\left(\alpha_{1}, \ldots, \alpha_{n-1}\right)$ over integer coefficients. This result will complete a circle of ideas initiated in [11] by Ishida. We also give some partial affirmative remarks toward the assertion that under certain condition our main result still holds to be true for two Bott manifolds just diffeomorphic, but not necessarily isomorphic, to each other.
\end{abstract}

\section{Introduction and main results}

Our main concern of this paper is a family of compact complex manifolds, called a Bott tower introduced first by Bott and Samelson in [2], which have recently attracted a great amount of attention in toric topology world (refer to [4] and [8]). They form a very nice family of toric manifolds, and possess several extra structures such as iterated fibrations and certain distinguished sections.

In order to construct such a family of compact complex manifolds, let $\mathbb{L}_{1}$ be a holomorphic complex line bundle over $B_{1}=\mathbb{C P}^{1}$. Then take its direct sum with the trivial complex line bundle $\mathbb{C}$ and projectivize each fiber to obtain a complex manifold $B_{2}=\mathbb{P}\left(\mathbb{C} \oplus \mathbb{L}_{1}\right)$. $\bar{B}_{2}$ is a fiber bundle over $B_{1}$ with a fiber $\mathbb{C P}^{1}$, and is called a Hirzebruch surface. We can repeat this process $n$ times, so that each $B_{j}$ is a fiber bundle over $B_{j-1}$ with a fiber $\mathbb{C P}^{1}$.

Received December 25, 2014; Revised July 22, 2015

2010 Mathematics Subject Classification. 57R19, 17R20, 57R25, 14M25.

Key words and phrases. Bott towers, Bott manifolds, Hirzebruch surfaces, toric varieties, Petrie's conjecture, strong cohomological rigidity conjecture. 
To be more precise, a Bott tower $\left\{\left(B_{j}\left(\alpha_{1}, \ldots, \alpha_{j}\right), \pi_{j}\right)\right\}_{j=1}^{n}$ of height $n$ is inductively defined as a sequence of $\mathbb{C P}^{1}$-bundles starting from a point $*$ :

$$
B_{n}\left(\alpha_{1}, \ldots, \alpha_{n}\right) \stackrel{\pi_{n}}{\longrightarrow} B_{n-1}\left(\alpha_{1}, \ldots, \alpha_{n-1}\right) \stackrel{\pi_{n-1}}{\longrightarrow} \cdots \stackrel{\pi_{2}}{\longrightarrow} B_{1}\left(\alpha_{1}\right) \stackrel{\pi_{1}}{\longrightarrow} B_{0}=\{*\},
$$

where $B_{j}\left(\alpha_{1}, \alpha_{2}, \ldots, \alpha_{j}\right)$ denotes the projectivization $\mathbb{P}\left(\underline{\mathbb{C}} \oplus \mathbb{L}_{j}\right)$ of the trivial complex line bundle $\underline{\mathbb{C}}$ and a complex line bundle $\mathbb{L}_{j}$ over $B_{j-1}\left(\alpha_{1}, \ldots, \alpha_{j-1}\right)$ with the first Chern class $\alpha_{j}$, and

$$
\pi_{j}: B_{j}\left(\alpha_{1}, \alpha_{2}, \ldots, \alpha_{j}\right) \rightarrow B_{j-1}\left(\alpha_{1}, \alpha_{2}, \ldots, \alpha_{j-1}\right)
$$

denotes the projection for each $j$ with $1 \leq j \leq n$. By definition, each $B_{j}\left(\alpha_{1}\right.$, $\left.\ldots, \alpha_{j}\right)$ is a toric manifold which admits an effective algebraic action of the torus $(\mathbb{C}-\{0\})^{n}$ having it as an open dense orbit, and it is called a $j$-step Bott manifold, or just a Bott manifold (refer to [2] and [9] for more details). Note that by its construction $\alpha_{1}$ always vanishes. Moreover, if all the first Chern classes $\alpha_{i}$ used to construct a Bott tower of height $n$ are zero, then $B_{n}\left(\alpha_{1}, \alpha_{2}, \ldots, \alpha_{n}\right)$ is diffeomorphic to $\left(\mathbb{C P}^{1}\right)^{n}$, and $B_{2}\left(\alpha_{1}, \alpha_{2}\right)$ is simply a Hirzebruch surface. Analogously, a generalized Bott manifold of height $n$ can be inductively defined also as a sequence of $\mathbb{C P}^{n_{i}}$-bundles with $n_{i} \geq 1$ starting from a point.

From now on, for the sake of simplicity, we will very often use the notation $B_{n}$ for $B_{n}\left(\alpha_{1}, \alpha_{2}, \ldots, \alpha_{n}\right)$, if there is no danger of any confusion. We shall also use the prime notation to denote any objects for the second Bott manifold $B_{n}\left(\alpha_{1}^{\prime}, \alpha_{2}^{\prime}, \ldots, \alpha_{n}^{\prime}\right)$.

Let $x_{j}$ denote the first Chern class of the tautological line bundle $\gamma_{j}$ over $B_{j}$. It follows from a formula of Borel and Hirzebruch in [1] that $H^{*}\left(B_{j} ; \mathbb{Z}\right)$ is a free module over $H^{*}\left(B_{j-1} ; \mathbb{Z}\right)$ through the projection $\pi_{j}^{*}: H^{*}\left(B_{j-1} ; \mathbb{Z}\right) \rightarrow$ $H^{*}\left(B_{j} ; \mathbb{Z}\right)$ with two generators 1 and $x_{j}$ of degree 0 and 2 , respectively. Thus, when $H^{*}\left(B_{j} ; \mathbb{Z}\right)$ is regarded as a subring of $H^{*}\left(B_{n} ; \mathbb{Z}\right)$ by using the pullback of the composition

$$
\pi_{j+1} \circ \pi_{j+2} \circ \cdots \circ \pi_{n}: B_{n} \stackrel{\pi_{n}}{\longrightarrow} B_{n-1} \stackrel{\pi_{n-1}}{\longrightarrow} \cdots \stackrel{\pi_{j+2}}{\longrightarrow} B_{j+1} \stackrel{\pi_{j+1}}{\longrightarrow} B_{j}
$$

of the projection maps $\pi_{j+1}, \pi_{j+2}, \ldots$, and $\pi_{n}$, it can be shown that the cohomology ring $H^{*}\left(B_{n} ; \mathbb{Z}\right)$ of $B_{n}\left(\alpha_{1}, \alpha_{2}, \ldots, \alpha_{n}\right)$ is given by

$$
\mathbb{Z}\left[x_{1}, x_{2}, \ldots, x_{n}\right] /\left\langle x_{i}^{2}=\alpha_{i} x_{i} \mid i=1,2, \ldots, n\right\rangle .
$$

Since $\alpha_{j} \in H^{2}\left(B_{j-1}\left(\alpha_{1}, \ldots, \alpha_{j-1}\right) ; \mathbb{Z}\right)$, it follows from [7], Proposition 3.1 that we may write

$$
\alpha_{j}=\sum_{i=1}^{j-1} c_{j}^{i} x_{i}, \quad c_{j}^{i} \in \mathbb{Z} .
$$

Note also that $H^{2}\left(B_{j} ; \mathbb{Z}\right)$ is isomorphic to $\mathbb{Z}^{j}$ and that $\mathbb{Z}^{j}$ is bijective with the collection of isomorphism classes of holomorphic line bundles over $B_{j}$ which are in turn classified by the first Chern classes ([9], Lemmas 2.11 and 2.14). 
For the purposes of this paper, an isomorphism between two Bott towers is defined to be a collection $\left\{F_{j}\right\}_{j=1}^{n}$ of diffeomorphisms

$$
F_{j}: B_{j}\left(\alpha_{1}, \alpha_{2}, \ldots, \alpha_{j-1}, \alpha_{j}\right) \rightarrow B_{j}\left(\alpha_{1}^{\prime}, \alpha_{2}^{\prime}, \ldots, \alpha_{j-1}^{\prime}, \alpha_{j}^{\prime}\right)
$$

which commute with the projection maps $\pi_{j}$ and $\pi_{j}^{\prime}$ in that the following diagram commutes:

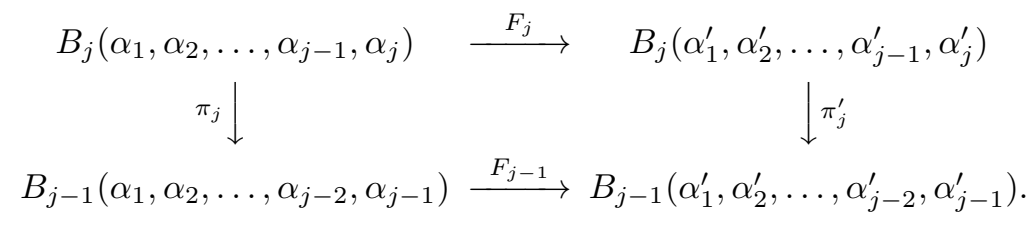

Note that this definition is weaker than that given in [9], Definition 2.6.

It is well-known from a result of Hirzebruch in $[10]^{1}$ that $B_{2}\left(\alpha_{1}, \alpha_{2}\right)$ is diffeomorphic to $\mathbb{C P}^{1} \times \mathbb{C P}^{1}$, if $\alpha_{2} \equiv 0 \bmod 2$, while $B_{2}\left(\alpha_{1}, \alpha_{2}\right)$ is diffeomorphic to $\mathbb{C P}^{1} \# \overline{\mathbb{C P}^{1}}$, otherwise. That is, we have the following theorem (see, e.g., [16], p. 16 or [14], Example 2.2).

Theorem 1.1. $B_{2}\left(\alpha_{1}, \alpha_{2}\right)$ is isomorphic (or diffeomorphic) to $B_{2}\left(\alpha_{1}, \alpha_{2}^{\prime}\right)$ if and only if $\alpha_{2} \equiv \alpha_{2}^{\prime} \bmod 2$ holds.

In particular, this implies that $H^{*}\left(B_{2}\left(\alpha_{1}, \alpha_{2}\right) ; \mathbb{Z}\right)$ is graded ring isomorphic to $H^{*}\left(B_{2}\left(\alpha_{1}, \alpha_{2}^{\prime}\right) ; \mathbb{Z}\right)$ if and only if both $\alpha_{2}$ and $\alpha_{2}^{\prime}$ are equal to $0 \bmod 2$, or equivalently $H^{*}\left(B_{2}\left(\alpha_{1}, \alpha_{2}\right) ; \mathbb{Z}\right)$ is graded ring isomorphic to $H^{*}\left(B_{2}\left(\alpha_{1}, \alpha_{2}^{\prime}\right) ; \mathbb{Z}\right)$ if and only if neither $\alpha_{2}$ nor $\alpha_{2}^{\prime}$ are equal to $0 \bmod 2$.

Related to these observations, the following conjecture has been posed (refer to [14] and [15]).

\section{Conjecture 1.2. Let}

$$
\psi: H^{*}\left(B_{n}\left(\alpha_{1}, \alpha_{2}, \ldots, \alpha_{n}\right) ; \mathbb{Z}\right) \rightarrow H^{*}\left(B_{n}\left(\alpha_{1}^{\prime}, \alpha_{2}^{\prime}, \ldots, \alpha_{n}^{\prime}\right) ; \mathbb{Z}\right)
$$

be a graded ring isomorphism over integers. Then $\psi$ is actually given by the pullback of a diffeomorphism from $B_{n}\left(\alpha_{1}^{\prime}, \alpha_{2}^{\prime}, \ldots, \alpha_{n}^{\prime}\right)$ to $B_{n}\left(\alpha_{1}, \alpha_{2}, \ldots, \alpha_{n}\right)$.

This conjecture is usually called a strong cohomological rigidity conjecture for Bott manifolds. It is still open in its full generality, and seems to be a very delicate and also difficult problem, even though there are some partial affirmative answers for certain special cases (refer to, e.g., [5], [6], and [13]). Conjecture 1.2 is also closely related to the well-known Petrie's conjecture, and there are some recent results for Bott manifolds, related to the Petrie's conjecture (see, e.g., [6], [12], [17] and [18]).

Our primary aim of this paper is not to directly deal with Conjecture 1.2 , but rather to generalize the theorem of Hirzebruch for the complex 2-dimensional Bott manifolds $B_{2}\left(\alpha_{1}, \alpha_{2}\right)$ to more general Bott towers of height $n$. In view of

\footnotetext{
${ }^{1}$ It needs to be remarked that the paper [10] contains many more interesting results other than this.
} 
the very definitions of Bott towers or Bott manifolds, we think that this kind of generalization is more appropriate and more useful in understanding the Bott towers or Bott manifolds.

One of the key problems which will play an important role in resolving the above conjecture is to classify complex vector bundles of rank two over a Bott manifold. Our first main result is to give a complete classification of those rank two complex vector bundles over a Bott manifold in terms of the total Chern classes, as follows.

Theorem 1.3. Let $\mathbb{E}_{1}$ and $\mathbb{E}_{2}$ be two complex vector bundles of rank 2 over a Bott manifold $B_{j}(j \geq 2)$ such that the total Chern class $c\left(\mathbb{E}_{1}\right)$ coincides with the total Chern class $c\left(\mathbb{E}_{2}\right)$. Then $\mathbb{E}_{1}$ is isomorphic to $\mathbb{E}_{2}$ as complex vector bundles.

Theorem 1.3 extends a result of Ishida in [11] where he proved Theorem 1.3 only for decomposable vector bundles of rank two over a Bott manifold. Note, however, that our proof is completely different from that of [11], Theorem 3.1.

As an application of Theorem 1.3, we can give the following theorem for Bott towers.

Theorem 1.4. Let a Bott tower $\left\{\left(B_{j}\left(\alpha_{1}, \ldots, \alpha_{j}\right), \pi_{j}\right)\right\}_{j=1}^{n-1}$ be isomorphic to

$$
\left\{\left(B_{j}\left(\alpha_{1}^{\prime}, \ldots, \alpha_{j}^{\prime}\right), \pi_{j}^{\prime}\right)\right\}_{j=1}^{n-1}
$$

by a family $\left\{F_{j}\right\}_{j=1}^{n-1}$ of diffeomorphisms, and let $\alpha_{n}$ and $\alpha_{n}^{\prime}$ be two elements of

$$
H^{2}\left(B_{n-1}\left(\alpha_{1}, \ldots, \alpha_{n-1}\right) ; \mathbb{Z}\right) \text { and } H^{2}\left(B_{n-1}\left(\alpha_{1}^{\prime}, \ldots, \alpha_{n-1}^{\prime}\right) ; \mathbb{Z}\right),
$$

respectively. Then the following three statements are equivalent.

(a) $\left(B_{n}\left(\alpha_{1}, \ldots, \alpha_{n-1}, \alpha_{n}\right), \pi_{n}\right)$ is diffeomorphic to $\left(B_{n}\left(\alpha_{1}^{\prime}, \ldots, \alpha_{n-1}^{\prime}, \alpha_{n}^{\prime}\right)\right.$, $\left.\pi_{n}^{\prime}\right)$ by a diffeomorphism $F_{n}$ which commutes with $\pi_{n}$ and $\pi_{n}^{\prime}$, so that two Bott towers $\left\{\left(B_{j}\left(\alpha_{1}, \alpha_{2}, \ldots, \alpha_{j}\right), \pi_{j}\right)\right\}_{j=1}^{n}$ and

$$
\left\{\left(B_{j}\left(\alpha_{1}^{\prime}, \alpha_{2}^{\prime}, \ldots, \alpha_{j}^{\prime}\right), \pi_{j}^{\prime}\right)\right\}_{j=1}^{n-1} \cup\left\{\left(B_{n}\left(\alpha_{1}^{\prime}, \alpha_{2}^{\prime}, \ldots, \alpha_{n-1}^{\prime}, \alpha_{n}^{\prime}\right), \pi_{n}^{\prime}\right)\right\}
$$

are isomorphic to each other.

(b) Two cohomology rings

$$
H^{*}\left(B_{n}\left(\alpha_{1}, \ldots, \alpha_{n}\right) ; \mathbb{Z}\right) \text { and } H^{2}\left(B_{n}\left(\alpha_{1}^{\prime}, \ldots, \alpha_{n}^{\prime}\right) ; \mathbb{Z}\right),
$$

as $H^{*}\left(B_{n-1} ; \mathbb{Z}\right)$-algebras are isomorphic to each other by the isomorphism $F_{n}^{*}$.

(c) Two elements $\alpha_{n}$ and $\alpha_{n}^{\prime}$ satisfy both conditions:

$$
\alpha_{n} \equiv F_{n-1}^{*}\left(\alpha_{n}^{\prime}\right) \bmod 2 \text { and } \alpha_{n}^{2}=\left(F_{n-1}^{*}\left(\alpha_{n}^{\prime}\right)\right)^{2} .
$$

Remark 1.5. (a) Since $F_{n-1}^{*}$ is a graded ring isomorphism, the condition that $\alpha_{n} \equiv F_{n-1}^{*}\left(\alpha_{n}^{\prime}\right) \bmod 2$ is equivalent to saying that either both of $\alpha_{2}$ and $\alpha_{2}^{\prime}$ are equal to $0 \bmod 2$ or neither of $\alpha_{2}$ and $\alpha_{2}^{\prime}$ are equal to 0 mod 2. This fits with the condition in Theorem 1.1. 
(b) Ishida also proved in [11], Corollary 4.3 that Theorem 1.4(a) is equivalent to Theorem $1.4(\mathrm{~b})$ by a conceptually similar but technically different method.

As an immediate consequence of Theorem 1.4, we have the following corollary which can be regarded as a higher dimensional analogue of a result of Hirzebruch in [10] (Theorem 1.1).

Corollary 1.6. Given two Bott manifolds

$$
B_{n}\left(\alpha_{1}, \alpha_{2}, \ldots, \alpha_{n-1}, \alpha_{n}\right) \text { and } B_{n}\left(\alpha_{1}, \alpha_{2}, \ldots, \alpha_{n-1}, \alpha_{n}^{\prime}\right),
$$

$B_{n}\left(\alpha_{1}, \ldots, \alpha_{n-1}, \alpha_{n}\right)$ is isomorphic to $B_{n}\left(\alpha_{1}, \ldots, \alpha_{n-1}, \alpha_{n}^{\prime}\right)$ as Bott towers if and only if both

$$
\alpha_{n} \equiv \alpha_{n}^{\prime} \bmod 2, \quad \text { and } \quad \alpha_{n}^{2}=\left(\alpha_{n}^{\prime}\right)^{2}
$$

hold.

Remark 1.7. In case of Hirzebruch surfaces, every complex vector bundle of rank 2 over $B_{1}\left(\alpha_{1}\right)=\mathbb{C P}^{1}$ is classified by the first Chern class only, so we do not need the second condition $\alpha_{2}^{2}=\left(\alpha_{2}^{\prime}\right)^{2}$ in Theorem 1.1, contrary to Theorem 1.3 or Corollary 1.6. In fact, in this case the extra condition $\alpha_{2}^{2}=\left(\alpha_{2}^{\prime}\right)^{2}$ automatically holds, since they always vanish, due to the degree reason.

Next we provide infinitely many non-trivial examples which satisfy two conditions in (1.2).

Example 1.8. Consider the 2-step Bott manifold $B_{2}\left(\alpha_{1}, \alpha_{2}\right)$ with $c_{2}^{1}=-(l+1)$ for any integer $l$. Let $\alpha_{3}$ and $\alpha_{3}^{\prime}$ be two elements of degree 2 of

$$
H^{*}\left(B_{2}\left(\alpha_{1}, \alpha_{2}\right) ; \mathbb{Z}\right)=\mathbb{Z}\left[x_{1}, x_{2}\right] /\left\langle x_{1}^{2}=0, x_{2}^{2}=\alpha_{2} x_{2}\right\rangle, \quad \alpha_{2}=c_{2}^{1} x_{1}
$$

such that

$$
\alpha_{3}=x_{1}+l x_{2}, \quad \text { and } \quad \alpha_{3}^{\prime}=(1+2 l) x_{1}+(l+2) x_{2} .
$$

Then clearly $\alpha_{3} \equiv \alpha_{3}^{\prime} \bmod 2$, and it is also easy to show that

$$
\alpha_{3}^{2}=-l(l-1)(l+2) x_{1} x_{2}=\left(\alpha_{3}^{\prime}\right)^{2},
$$

as required. Therefore, in this case two Bott manifolds

$$
B_{3}\left(\alpha_{1}, \alpha_{2}, \alpha_{3}\right) \text { and } B_{3}\left(\alpha_{1}, \alpha_{2}, \alpha_{3}^{\prime}\right)
$$

are isomorphic to each other by Corollary 1.6.

It is a natural question to ask whether or not Theorem 1.4 or Corollary 1.6 is also true just for two diffeomorphic, but not necessarily isomorphic, Bott manifolds, at least up to some permutation in the symmetric group $S_{n}$ on the index set $\{1,2, \ldots, n\}$. In Section 4 , we give some affirmative remark for this question, as follows (refer to Section 4, Lemma 4.4 for more on the notation $\left.r_{i}\right)$. 
Theorem 1.9. Let $B_{n}\left(\alpha_{1}, \ldots, \alpha_{n}\right)$ be diffeomorphic to $B_{n}\left(\alpha_{1}^{\prime}, \ldots, \alpha_{n}^{\prime}\right)$ by a diffeomorphism $F$, and let

$$
\psi: H^{*}\left(B_{n}\left(\alpha_{1}, \alpha_{2}, \ldots, \alpha_{n}\right) ; \mathbb{Z}\right) \rightarrow H^{*}\left(B_{n}\left(\alpha_{1}^{\prime}, \alpha_{2}^{\prime}, \ldots, \alpha_{n}^{\prime}\right) ; \mathbb{Z}\right)
$$

be the graded ring isomorphism over integers given by the pullback of $F^{-1}$. If all of $r_{i}(1 \leq i \leq n)$ are equal to \pm 1 , then there is some permutation $\sigma \in S_{n}$ such that we have

for all $1 \leq i \leq n$.

$$
\psi\left(\alpha_{i}\right) \equiv \alpha_{\sigma(i)}^{\prime} \bmod 2
$$

We organize this paper, as follows. In Section 2, we prove an important result that all complex vector bundles of rank 2 over a Bott manifold are classified by their total Chern classes (Theorem 2.1). In Section 3, we give a detailed proof of Theorem 1.4 by essentially using the mathematical induction on $n$. In this section, we give a proof of a simpler version of Theorem 1.4 which is easily shown to imply the full version of Theorem 1.4. Section 4 is devoted to the proof of Theorem 1.9 as well as some other related discussions.

\section{Proof of Theorem 1.3}

The aim of this section is to give a proof of Theorem 1.3 which will be crucially used in the proof of Theorem 1.4. As mentioned in Section 1, this theorem says that all complex vector bundles of rank 2 over a Bott manifold are classified by their total Chern classes.

Theorem 2.1. Let $\mathbb{E}_{1}$ and $\mathbb{E}_{2}$ be two complex vector bundles of rank 2 over a Bott manifold $B_{j}(j \geq 2)$. Then $\mathbb{E}_{1}$ is isomorphic to $\mathbb{E}_{2}$ as complex vector bundles if and only if the total Chern class $c\left(\mathbb{E}_{1}\right)$ coincides with the total Chern class $c\left(\mathbb{E}_{2}\right)$.

Proof. To prove it, note first from [5], Lemma 3.4 that every complex vector bundle of rank 2 over a Bott manifold $B_{2}$ is classified by the total Chern class. Thus we need to prove the theorem only for $j \geq 3$. To do so, consider the following exact sequence

$$
\left[B_{j}, U(\infty) / U(2)\right] \rightarrow\left[B_{j}, B U(2)\right] \stackrel{c}{\rightarrow}\left[B_{j}, B U(\infty)\right]
$$

induced from the fibration

$$
U(\infty) / U(2) \rightarrow B U(2) \rightarrow B U(\infty),
$$

where $U(\infty)=\cup_{n=1}^{\infty} U(n)$ is obtained as the telescoping construction given by the standard embedding (see, e.g., [3], p.241). It can be shown as in [5], Lemma 3.4 that

$$
\left[B_{1}, U(\infty) / U(2)\right]=\left[B_{2}, U(\infty) / U(2)\right]=0,
$$

since $B_{1}$ and $B_{2}$ are of the real dimension at most 4 , and $U / U(2)$ is 4 -connected. Recall that $U(\infty) / U(2)$ is 4-connected, since $U(3) / U(2)$ is diffeomorphic to $S^{5}$ and $U(3) / U(2)$ is embedded into $U(\infty) / U(2)$ as a telescoping subspace in the natural way (see, e.g., [19], p. 127). 
Note also that there is an exact sequence

$$
\left[B_{j-1}, U(\infty) / U(2)\right] \rightarrow\left[B_{j}, U(\infty) / U(2)\right] \rightarrow\left[B_{1}, U(\infty) / U(2)\right]
$$

induced from the fibration

$$
B_{1}=\mathbb{C P}^{1} \hookrightarrow B_{j} \rightarrow B_{j-1} .
$$

Since $\left[B_{1}, U(\infty) / U(2)\right]=\left[B_{2}, U(\infty) / U(2)\right]=0$, it follows inductively from the exact sequence $(2.2)$ that all $\left[B_{j}, U(\infty) / U(2)\right]=0$ for all $j \geq 3$. Thus, by the exact sequence (2.1) the Chern class map $c$ is injective.

Since $H^{\text {odd }}\left(B_{j} ; \mathbb{Z}\right)=0,\left[B_{j}, B U(\infty)\right]$ is torsion free. This together with the fact that $c$ is injective implies that all elements of $\left[B_{j}, B U(2)\right]$ can be classified by their Chern classes. This completes the proof of Theorem 2.1.

\section{Proof of Theorem 1.4}

The aim of this section is to give a proof of Theorem 1.3. To do so, we begin with the following theorem which clearly proves that Theorem 1.4(c) implies Theorem 1.4(a).

Theorem 3.1. Let a Bott tower $\left\{\left(B_{j}\left(\alpha_{1}, \ldots, \alpha_{j}\right), \pi_{j}\right)\right\}_{j=1}^{n-1}$ be isomorphic to itself by a family $\left\{F_{j}\right\}_{j=1}^{n-1}$ of diffeomorphisms, and let $\alpha_{n}$ and $\alpha_{n}^{\prime}$ be two elements of $H^{2}\left(B_{n-1}\left(\alpha_{1}, \ldots, \alpha_{n-1}\right) ; \mathbb{Z}\right)$ such that

(a) $\alpha_{n} \equiv F_{n-1}^{*}\left(\alpha_{n}^{\prime}\right) \bmod 2$, and

(b) $\alpha_{n}^{2}=\left(F_{n-1}^{*}\left(\alpha_{n}^{\prime}\right)\right)^{2}$.

Then $\left(B_{n}\left(\alpha_{1}, \ldots, \alpha_{n-1}, \alpha_{n}\right), \pi_{n}\right)$ is diffeomorphic to $\left(B_{n}\left(\alpha_{1}, \ldots, \alpha_{n-1}, \alpha_{n}^{\prime}\right), \pi_{n}^{\prime}\right)$ by a diffeomorphism $F_{n}$ which commutes with $\pi_{n}$ and $\pi_{n}^{\prime}$, so that two Bott towers $\left\{\left(B_{j}\left(\alpha_{1}, \alpha_{2}, \ldots, \alpha_{j}\right), \pi_{j}\right)\right\}_{j=1}^{n}$ and

$$
\left\{\left(B_{j}\left(\alpha_{1}, \alpha_{2}, \ldots, \alpha_{j}\right), \pi_{j}\right)\right\}_{j=1}^{n-1} \cup\left\{\left(B_{n}\left(\alpha_{1}, \alpha_{2}, \ldots, \alpha_{n-1}, \alpha_{n}^{\prime}\right), \pi_{n}^{\prime}\right)\right\}
$$

are isomorphic to each other.

Proof. To prove it, we first assume that both $\alpha_{n} \equiv F_{n-1}^{*}\left(\alpha_{n}^{\prime}\right) \bmod 2$ and $\alpha_{n}^{2}=$ $\left(F_{n-1}^{*}\left(\alpha_{n}^{\prime}\right)\right)^{2}$ hold in $H^{2}\left(B_{n-1}\left(\alpha_{1}, \ldots, \alpha_{n-1}\right) ; \mathbb{Z}\right)$, and then use the induction on $n$. Note that Theorem 3.1 holds for $n=2$ case, due to the validity of Theorem 1.1 .

So suppose that Theorem 3.1 holds for any $n-1 \geq 2$. Then consider the following commutative diagram:

$$
\begin{array}{ccc}
B_{n}\left(\alpha_{1}, \alpha_{2}, \ldots, \alpha_{n-1}, F_{n-1}^{*}\left(\alpha_{n}^{\prime}\right)\right) \stackrel{\tilde{F}_{n}}{\longrightarrow} & B_{n}\left(\alpha_{1}, \alpha_{2}, \ldots, \alpha_{n-1}, \alpha_{n}^{\prime}\right) \\
\tilde{\pi}_{n} \downarrow & \downarrow \pi_{n}^{\prime} \\
B_{n-1}\left(\alpha_{1}, \alpha_{2}, \ldots, \alpha_{n-2}, \alpha_{n-1}\right) \stackrel{F_{n-1}}{\longrightarrow} B_{n-1}\left(\alpha_{1}, \alpha_{2}, \ldots, \alpha_{n-2}, \alpha_{n-1}\right) .
\end{array}
$$

Here the Bott manifold $B_{n}\left(\alpha_{1}, \alpha_{2}, \ldots, \alpha_{n-1}, F_{n-1}^{*}\left(\alpha_{n}^{\prime}\right)\right)$ is nothing but the projectivization $\mathbb{P}\left(\underline{\mathbb{C}} \oplus \tilde{\mathbb{L}}_{n}\right)$ of the trivial complex line bundle $\underline{\mathbb{C}}$ and a complex line bundle $\tilde{\mathbb{L}}_{n}$ with the first Chern class $F_{n-1}^{*}\left(\alpha_{n}^{\prime}\right)$ over $B_{n-1}\left(\alpha_{1}, \ldots, \alpha_{n-1}\right)$, and $\tilde{F}_{n}$ 
is a bundle isomorphism between $B_{n}\left(\alpha_{1}, \ldots, \alpha_{n-1}, F_{n-1}^{*}\left(\alpha_{n}^{\prime}\right)\right)$ and $B_{n}\left(\alpha_{1}, \ldots\right.$, $\left.\alpha_{n-1}, \alpha_{n}^{\prime}\right)$. Thus, in particular, $B_{n}\left(\alpha_{1}, \ldots, \alpha_{n-1}, F_{n-1}^{*}\left(\alpha_{n}^{\prime}\right)\right)$ and $B_{n}\left(\alpha_{1}, \ldots\right.$, $\left.\alpha_{n-1}, \alpha_{n}^{\prime}\right)$ are diffeomorphic to each other by $\tilde{F}_{n}$.

Next we want to show that $B_{n}\left(\alpha_{1}, \alpha_{2}, \ldots, \alpha_{n-1}, F_{n-1}^{*}\left(\alpha_{n}^{\prime}\right)\right)$ is actually diffeomorphic to $B_{n}\left(\alpha_{1}, \alpha_{2}, \ldots, \alpha_{n-1}, \alpha_{n}\right)$ by a diffeomorphism $G$ which commutes with $\pi_{n}$ and $\tilde{\pi}_{n}$. To do so, note first that, since $F_{n-1}^{*}\left(\alpha_{n}^{\prime}\right) \equiv \alpha_{n} \bmod 2$, there are some integers $b_{n}^{j}$ such that

$$
F_{n-1}^{*}\left(\alpha_{n}^{\prime}\right)=\alpha_{n}+2 \sum_{j=1}^{n-1} b_{n}^{j} x_{j} .
$$

We then recall the following well-known fact (refer to, e.g., [5], Lemma 2.1).

Lemma 3.2. Let $\pi: \mathbb{E} \rightarrow B$ be a complex vector bundle over a smooth manifold $B$ and let $\mathbb{P}(\mathbb{E})$ be the projectivization of $E$. Let $\mathbb{L}$ be a complex line bundle over $B$. We denote by $\mathbb{E}^{*}$ the complex vector bundle dual to $\mathbb{E}$. Then both $\mathbb{P}\left(\mathbb{E}^{*}\right)$ and $\mathbb{P}(\mathbb{E} \otimes \mathbb{L})$ are isomorphic to $\mathbb{P}(\mathbb{E})$ as fiber bundles, and, in particular, they are diffeomorphic to each other.

Let $\gamma$ be a complex line bundle over $B_{n-1}\left(\alpha_{1}, \ldots, \alpha_{n-1}\right)$ defined by

$$
\gamma=\gamma_{1}^{-b_{n}^{1}} \otimes \gamma_{2}^{-b_{n}^{2}} \otimes \cdots \otimes \gamma_{n-1}^{-b_{n}^{n-1}}
$$

where $\gamma_{j}$ denotes the tautological line bundle over $B_{j}$, and here is regarded as a complex line bundle over the same Bott manifold $B_{n-1}$ through the obvious pullback map.

Lemma 3.3. The first Chern class $c_{1}\left(\left(\underline{\mathbb{C}} \oplus \tilde{\mathbb{L}}_{n}\right) \otimes \gamma\right)$ of a complex vector bundle $\left(\underline{\mathbb{C}} \oplus \tilde{\mathbb{L}}_{n}\right) \otimes \gamma$ of rank 2 over $B_{n-1}\left(\alpha_{1}, \ldots, \alpha_{n-1}\right)$ is equal to $\alpha_{n}$ that is the first Chern class of a complex line bundle $\mathbb{L}_{n}$ over the same Bott manifold $B_{n-1}\left(\alpha_{1}, \ldots, \alpha_{n-1}\right)$.

Proof. It is easy to see

$$
\begin{aligned}
c_{1}\left(\left(\underline{\mathbb{C}} \oplus \tilde{\mathbb{L}}_{n}\right) \otimes \gamma\right) & =c_{1}(\gamma)+c_{1}\left(\tilde{\mathbb{L}}_{n} \otimes \gamma\right)=2 c_{1}(\gamma)+c_{1}\left(\tilde{\mathbb{L}}_{n}\right) \\
& =-2 \sum_{j=1}^{n-1} b_{n}^{j} x_{j}+F_{n-1}^{*}\left(\alpha_{n}^{\prime}\right) \stackrel{(3.1)}{=} \alpha_{n},
\end{aligned}
$$

as desired.

With the help of Theorem 2.1, we can prove the following corollary.

Corollary 3.4. Two complex vector bundles $\left(\underline{\mathbb{C}} \oplus \tilde{\mathbb{L}}_{n}\right) \otimes \gamma$ and $\underline{\mathbb{C}} \oplus \mathbb{L}_{n}$ of rank 2 over $B_{n-1}\left(\alpha_{1}, \ldots, \alpha_{n-1}\right)$ is isomorphic to each other, as complex vector bundles of rank 2 .

Proof. To prove it, recall first that by assumption we have the following identities:

$$
\alpha_{n}^{2}=\left(F_{n-1}^{*}\left(\alpha_{n}^{\prime}\right)\right)^{2}=c_{1}\left(\tilde{\mathbb{L}}_{n}\right)^{2} \text { in } H^{*}\left(B_{n-1} ; \mathbb{Z}\right) .
$$


Since by Lemma 3.3 we have

$$
\alpha_{n}=2 c_{1}(\gamma)+c_{1}\left(\tilde{\mathbb{L}}_{n}\right)
$$

and so

$$
\alpha_{n}^{2}=4 c_{1}(\gamma)^{2}+4 c_{1}(\gamma) c_{1}\left(\tilde{\mathbb{L}}_{n}\right)+c_{1}\left(\tilde{\mathbb{L}}_{n}\right)^{2},
$$

it follows from (3.2) that we have

$$
4\left(c_{1}(\gamma)^{2}+c_{1}(\gamma) c_{1}\left(\tilde{\mathbb{L}}_{n}\right)\right)=\alpha_{n}^{2}-c_{1}\left(\tilde{\mathbb{L}}_{n}\right)^{2}=0 \text { in } H^{*}\left(B_{n-1} ; \mathbb{Z}\right) .
$$

Since there is no torsion in $H^{*}\left(B_{n-1} ; \mathbb{Z}\right)$, this implies that

$$
c_{1}(\gamma)^{2}+c_{1}(\gamma) c_{1}\left(\tilde{\mathbb{L}}_{n}\right)=0
$$

It is also easy to show that in $H^{*}\left(B_{n-1} ; \mathbb{Z}\right)$ we have

$$
\begin{aligned}
c\left(\left(\underline{\mathbb{C}} \oplus \tilde{\mathbb{L}}_{n}\right) \otimes \gamma\right) & =1+c_{1}\left(\left(\underline{\mathbb{C}} \oplus \tilde{\mathbb{L}}_{n}\right) \otimes \gamma\right)+c_{2}\left(\left(\underline{\mathbb{C}} \oplus \tilde{\mathbb{L}}_{n}\right) \otimes \gamma\right) \\
& =c(\gamma) c\left(\tilde{\mathbb{L}}_{n} \otimes \gamma\right)=\left(1+c_{1}(\gamma)\right)\left(1+c_{1}\left(\tilde{\mathbb{L}}_{n}\right)+c_{1}(\gamma)\right) \\
& =1+\left(2 c_{1}(\gamma)+c_{1}\left(\tilde{\mathbb{L}}_{n}\right)\right)+\left(c_{1}(\gamma) c_{1}\left(\tilde{\mathbb{L}}_{n}\right)+c_{1}(\gamma)^{2}\right) \\
& =1+\alpha_{n}, \quad \text { by }(3.3) \text { and }(3.4) \\
& =c\left(\underline{\mathbb{C}} \oplus \mathbb{L}_{n}\right) .
\end{aligned}
$$

Therefore, it follows from Theorem 2.1 that two vector bundles $\left(\underline{\mathbb{C}} \oplus \tilde{\mathbb{L}}_{n}\right) \otimes \gamma$ and $\underline{\mathbb{C}} \oplus \mathbb{L}_{n}$ are isomorphic to each other, as claimed.

Finally, we are ready to finish the proof of Theorem 3.1, as follows. Since $B_{n}\left(\alpha_{1}, \ldots, \alpha_{n-1}, F_{n-1}^{*}\left(\alpha_{n}^{\prime}\right)\right)$ is diffeomorphic to $\mathbb{P}\left(\underline{\mathbb{C}} \oplus \tilde{\mathbb{L}}_{n}\right)$, it follows from Lemma 3.2 that it is also diffeomorphic to $\mathbb{P}\left(\left(\underline{\mathbb{C}} \oplus \tilde{\mathbb{L}}_{n}\right) \otimes \gamma\right)$. On the other hand, the total Chern class of $\left(\underline{\mathbb{C}} \oplus \tilde{\mathbb{L}}_{n}\right) \otimes \gamma$ coincides with that of $\underline{\mathbb{C}} \oplus \mathbb{L}_{n}$, and so they are isomorphic to each other by Corollary 3.4. Hence clearly their projectivizations $B_{n}\left(\alpha_{1}, \ldots, \alpha_{n-1}, F_{n-1}^{*}\left(\alpha_{n}^{\prime}\right)\right)$ and $B_{n}\left(\alpha_{1}, \ldots, \alpha_{n-1}, \alpha_{n}\right)$ should be diffeomorphic by a diffeomorphism $G$. Notice also that by its construction $G$ commutes with the projection maps $\pi_{n}$ and $\tilde{\pi}_{n}$, as follows.

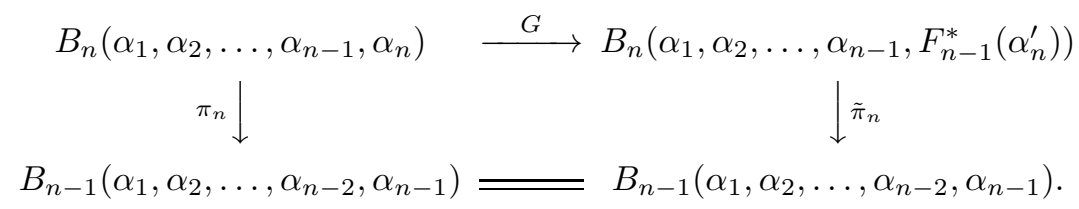

It is now easy to see that the composition $F_{n}:=\tilde{F}_{n} \circ G$ of two diffeomorphisms $G$ and $\tilde{F}_{n}$ gives rise to a diffeomorphism between two Bott manifolds $B_{n}\left(\alpha_{1}, \ldots, \alpha_{n-1}, \alpha_{n}\right)$ and $B_{n}\left(\alpha_{1}, \ldots, \alpha_{n-1}, \alpha_{n}^{\prime}\right)$ which commutes with $\pi_{n}$ and $\pi_{n}^{\prime}$. Therefore, two Bott towers $\left\{\left(B_{j}\left(\alpha_{1}, \ldots, \alpha_{j}\right), \pi_{j}\right)\right\}_{j=1}^{n}$ and

$$
\left\{\left(B_{j}\left(\alpha_{1}, \ldots, \alpha_{j}\right), \pi_{j}\right)\right\}_{j=1}^{n-1} \cup\left\{\left(B_{n}\left(\alpha_{1}, \alpha_{2}, \ldots, \alpha_{n-1}, \alpha_{n}^{\prime}\right), \pi_{n}^{\prime}\right)\right\}
$$

are isomorphic to each other. This implies that by the induction on $n$ Theorem 3.1 holds for any $n \geq 2$, which completes the proof. 
Note that clearly Theorem 1.4(a) implies Theorem 1.4(b). Next we show that Theorem 1.4(b) implies Theorem 1.4(c), as follows.

Theorem 3.5. Assume that two cohomology rings

$$
H^{*}\left(B_{n}\left(\alpha_{1}, \ldots, \alpha_{n-1}, \alpha_{n}\right) ; \mathbb{Z}\right) \text { and } H^{*}\left(B_{n}\left(\alpha_{1}, \ldots, \alpha_{n-1}, \alpha_{n}^{\prime}\right) ; \mathbb{Z}\right),
$$

as $H^{*}\left(B_{n-1} ; \mathbb{Z}\right)$-algebras are isomorphic to each other by the isomorphism $F_{n}^{*}$. Then two elements $\alpha_{n}$ and $\alpha_{n}^{\prime}$ satisfy both conditions:

$$
\alpha_{n} \equiv F_{n-1}^{*}\left(\alpha_{n}^{\prime}\right) \bmod 2 \text { and } \alpha_{n}^{2}=\left(F_{n-1}^{*}\left(\alpha_{n}^{\prime}\right)\right)^{2} .
$$

Proof. For simplicity, let $\Psi=F_{n}^{*}, x=x_{n}$, and $y=x_{n}^{\prime}$. To prove the theorem, note also that it suffices to prove it under the assumption that $F_{n-1}^{*}$ is the identity map.

To begin with the proof, we let

$$
\Psi(x)= \pm y+\beta, \quad \beta \in H^{*}\left(B_{n-1} ; \mathbb{Z}\right) .
$$

Since $\Psi\left(x^{2}-\alpha_{n} x\right)=0$ and $y^{2}-\alpha_{n}^{\prime} y=0$, we have

$$
\begin{aligned}
0 & =\Psi\left(x^{2}-\alpha_{n} x\right)=\Psi(x)^{2}-\alpha_{n} \Psi(x)=( \pm y+\beta)^{2}-\alpha_{n}( \pm y+\beta) \\
& =\left(\alpha_{n}^{\prime} \mp \alpha_{n} \pm 2 \beta\right) y+\left(\beta^{2}-\alpha_{n} \beta\right) .
\end{aligned}
$$

Thus we obtain

$$
\alpha_{n}^{\prime} \mp \alpha_{n} \pm 2 \beta=0 \text { and } \beta^{2}-\alpha_{n} \beta=0 .
$$

The first equation of (3.5) implies that we have

$$
\alpha_{n}^{\prime} \equiv \alpha_{n} \bmod 2 .
$$

On the other hand, by plugging $2 \beta=\alpha_{n} \pm \alpha_{n}^{\prime}$ into the second equation $\beta^{2}-$ $\alpha_{n} \beta=0$ of (3.5), it is easy to see that we have $\alpha_{n}^{2}=\left(\alpha_{n}^{\prime}\right)^{2}$, as desired.

\section{Further remarks}

As mentioned in Section 1, it is a natural question to ask whether or not Theorem 1.3 or Corollary 1.6 still holds for two diffeomorphic Bott manifolds by a more general diffeomorphism which does not necessarily come from Bott towers. The aim of this section is to discuss this question in more detail.

To do so, we first want to recall an alternative construction of Bott towers or Bott manifolds (see [9] for more details). It is easy to see that a one-step Bott tower $B_{1}\left(\alpha_{1}\right)=\mathbb{C P}^{1}$ can be obtained as a quotient of $\left(\mathbb{C}^{2}-\{0\}\right) / \mathbb{C}^{*}$, where $\mathbb{C}^{*}$ denotes the complex numbers $\mathbb{C}$ minus the origin, and $\mathbb{C}^{*}$ acts on $\left(\mathbb{C}^{*}\right)^{2}$ diagonally. Then take a complex line bundle $\mathbb{L}_{1}=\left(\mathbb{C}^{*}\right)^{2} \times_{\mathbb{C}^{*}} \mathbb{C}$ over $B_{1}$, where $g_{1} \in \mathbb{C}^{*}$ acts on $\mathbb{C}$ by $g_{1} \cdot v=g_{1}^{c_{2}^{1}} v$ for some $c_{2}^{1} \in \mathbb{Z}$ and in $\mathbb{L}_{1}$ we have

$$
\left[\left(z_{1}, w_{1}\right), v\right]=\left[\left(z_{1} g_{1}, w_{1} g_{1}\right), g_{1}^{-c_{2}^{1}} v\right]
$$


Let $\alpha_{2}=c_{1}\left(\mathbb{L}_{1}\right) \in H^{2}\left(B_{1}\left(\alpha_{1}\right) ; \mathbb{Z}\right)$. Then the 2-step Bott tower $B_{2}\left(\alpha_{1}, \alpha_{2}\right)=$ $\mathbb{P}\left(\underline{\mathbb{C}} \oplus \mathbb{L}_{1}\right)$ can be written as the quotient $\left(\mathbb{C}^{2}-\{0\}\right)^{2} /\left(\mathbb{C}^{*}\right)^{2}$, where $\left(g_{1}, g_{2}\right) \in$ $\left(\mathbb{C}^{*}\right)^{2}$ acts on $\left(\mathbb{C}^{2}-\{0\}\right)^{2}$ by

$$
\left(g_{1}, g_{2}\right) \cdot\left(\left(z_{1}, w_{1}\right),\left(z_{2}, w_{2}\right)\right)=\left(\left(z_{1} g_{1}, w_{1} g_{1}\right),\left(z_{2} g_{2}, g_{1}^{-c_{2}^{1}} w_{2} g_{2}\right)\right) .
$$

We can continue this process to construct higher dimensional Bott towers

$$
\left\{\left(B_{j}\left(\alpha_{1}, \ldots, \alpha_{j}\right), \pi_{j}\right)\right\}_{j=1}^{n}
$$

as the quotient of $\left(\mathbb{C}^{2}-\{0\}\right)^{n} /\left(\mathbb{C}^{*}\right)^{n}$ by the free action of $\left(\mathbb{C}^{*}\right)^{n}$ given by

$$
\begin{aligned}
& \left(g_{1}, g_{2}, \ldots, g_{j}, \ldots, g_{n}\right) \cdot\left(\left(z_{1}, w_{1}\right),\left(z_{2}, w_{2}\right), \ldots,\left(z_{j}, w_{j}\right), \ldots,\left(z_{n}, w_{n}\right)\right) \\
= & \left(\left(z_{1} g_{1}, w_{1} g_{1}\right),\left(z_{2} g_{2}, g_{1}^{-c_{2}^{1}} w_{2} g_{2}\right), \ldots,\right. \\
& \left.\left(z_{j} g_{j},\left(\prod_{i=1}^{j-1} g_{i}^{-c_{j}^{i}}\right) w_{j} g_{j}\right), \ldots,\left(w_{n} g_{n},\left(\prod_{i=1}^{n-1} g_{i}^{-c_{n}^{i}}\right) w_{n} g_{n}\right)\right),
\end{aligned}
$$

where $\left\{c_{j}^{i}\right\}_{1 \leq i<j \leq n}$ denotes any collection of $n(n-1) / 2$ integers. The obvious projections given by

$$
\begin{aligned}
\left(\left(z_{1}, w_{1}\right), \ldots,\left(z_{n}, w_{n}\right)\right) \stackrel{\pi_{n}}{\longrightarrow}\left(\left(z_{1}, w_{1}\right), \ldots,\left(z_{n-1}, w_{n-1}\right)\right) \stackrel{\pi_{n-1}}{\longrightarrow} \ldots \\
\ldots \stackrel{\pi_{3}}{\longrightarrow}\left(\left(z_{1}, w_{1}\right),\left(z_{2}, w_{2}\right)\right) \stackrel{\pi_{2}}{\longrightarrow}\left(z_{1}, w_{1}\right) \stackrel{\pi_{1}}{\longrightarrow}\{*\}
\end{aligned}
$$

then induces the Bott tower. As mentioned in Section 1, (1.1), it is interesting to note that

$$
\alpha_{j}=\sum_{i=1}^{j-1} c_{j}^{i} x_{i}, \quad c_{j}^{i} \in \mathbb{Z}
$$

Let $\mathcal{N}_{n}$ be the set of integral strictly upper triangular square matrices of size $n$. Then it has been shown in [9], Section 2.3 that the map from $\mathcal{N}_{n}$ to the collection of isomorphism classes of $n$-step Bott towers is bijective. In other words, the Bott tower is also determined by a matrix $C \in \mathcal{N}_{n}$, and so we may write the $n$-step Bott tower as $B_{n}(C)$ for some $C \in \mathcal{N}_{n}$. If $C$ is the zero matrix in $\mathcal{N}_{n}$, then clearly $B_{n}(C)$ is diffeomorphic to $\left(\mathbb{C P}^{1}\right)^{n}$, and the converse is also true $([14])$.

However, in general $B_{n}(C)$ and $B_{n}(D)$ may be diffeomorphic, even if $C$ and $D$ are different. In particular, let $P$ denote the permutation matrix of a permutation $\sigma$ in $S_{n}$ on $n$ letters $\{1,2, \ldots, n\}$ whose $(i, j)$-entry is 1 if $\sigma(j)=i$, and 0 otherwise. When both $C$ and $P C P^{-1}$ are elements of $\mathcal{N}_{n}, B_{n}(C)$ and $B_{n}\left(P C P^{-1}\right)$ are diffeomorphic (refer to [6], Lemma 6.1). In view of these observations, the converse of Corollary 1.6 might not be true in that form, but might be true at least up to some permutation of the index set $\{1,2, \ldots, n\}$.

For the rest of this section, we will make this discussion more precise and deal with a more general case of Theorem 1.3 and Corollary 1.6. To do so, we 
first need to collect some preliminary results. As in the paper [6], it will be useful to let

$$
y_{i}=x_{i}-\frac{1}{2} \alpha_{i}, \quad 1 \leq i \leq n .
$$

Then it is easy to see from (4.1) that the following lemma holds.

Lemma 4.1. For each $1 \leq i \leq n$, we have

$$
x_{i}=\sum_{j=1}^{i} b_{i}^{j} y_{j},
$$

where all of $b_{i}^{j}$ are elements of $\mathbb{Q}$ and $b_{i}^{i}=1$.

Proof. To prove it, we will use the mathematical induction on $n$. For $n=1$, clearly $x_{1}=y_{1}$, since $\alpha_{1}=0$. Next suppose that the lemma holds for any $n-1 \geq 1$. Then we have

$$
\begin{aligned}
x_{n} & =y_{n}+\frac{1}{2} \alpha_{n}=y_{n}+\frac{1}{2} \sum_{j=1}^{n-1} c_{n}^{j} x_{j}=y_{n}+\frac{1}{2} \sum_{j=1}^{n-1}\left(c_{n}^{j}\left(\sum_{k=1}^{j} b_{j}^{k} y_{k}\right)\right) \\
& =y_{n}+\frac{1}{2} \sum_{k=1}^{n-1}\left(\sum_{j=k}^{n-1} c_{n}^{j} b_{j}^{k}\right) y_{k},
\end{aligned}
$$

which completes the proof of the identity of the equation (4.2) for any $n$, as desired.

Note that Lemma 4.1 actually implies that $x_{1}, x_{2}, \ldots, x_{n}$ and $y_{1}, y_{2}, \ldots, y_{n}$ satisfy the following linear equation

$$
\left(\begin{array}{c}
x_{1} \\
x_{2} \\
x_{3} \\
x_{4} \\
\vdots \\
x_{n-1} \\
x_{n}
\end{array}\right)=\left(\begin{array}{ccccccc}
1 & 0 & 0 & 0 & \cdots & 0 & 0 \\
* & 1 & 0 & 0 & \cdots & 0 & 0 \\
* & * & 1 & 0 & \cdots & 0 & 0 \\
* & * & * & 1 & \cdots & 0 & 0 \\
\vdots & \vdots & \vdots & \vdots & \ddots & \vdots & \vdots \\
* & * & * & * & \cdots & 1 & 0 \\
* & * & * & * & \cdots & * & 1
\end{array}\right)_{n \times n} \quad\left(\begin{array}{c}
y_{1} \\
y_{2} \\
y_{3} \\
y_{4} \\
\vdots \\
y_{n-1} \\
y_{n}
\end{array}\right)
$$

Since the coefficient matrix of the above linear equation (4.3) is lower triangular and unipotent, we can also express $y_{1}, y_{2}, \ldots, y_{n}$ in terms of $x_{1}, x_{2}, \ldots, x_{n}$ in such a way that we have

$$
y_{i}=\sum_{j=1}^{i} \tilde{b}_{i}^{j} x_{j},
$$

where all of $\tilde{b}_{i}^{j}$ are elements of $\mathbb{Q}$ and $\tilde{b}_{i}^{i}=1$.

The following lemma immediately follows from (4.1), so that its proof will be left to the reader. 
Lemma 4.2. If $x_{i}^{2}=\alpha_{i} x_{i}$ holds, then $y_{i}^{2}=\frac{1}{4} \alpha_{i}^{2}$ holds. Conversely, if $y_{i}^{2}=\frac{1}{4} \alpha_{i}^{2}$ holds, then $x_{i}^{2}=\alpha_{i} x_{i}$ holds.

For the sake of notational simplicity, let

$$
\begin{aligned}
P & :=\mathbb{Q}\left[x_{1}, x_{2}, \ldots, x_{n}\right] /\left\langle x_{i}^{2}=\alpha_{i} x_{i} \mid i=1,2, \ldots, n\right\rangle, \\
Q & :=\mathbb{Q}\left[y_{1}, y_{2}, \ldots, y_{n}\right] /\left\langle y_{i}^{2}=\frac{1}{4} \alpha_{i}^{2} \mid i=1,2, \ldots, n\right\rangle .
\end{aligned}
$$

Then, it is straightforward to see from the identities (1.1) and (4.1), and Lemmas 4.1 and 4.2 that $P$ is isomorphic to $Q$. It is often convenient to use $Q$ instead of $P$ in order to show some important properties of the cohomology ring $H^{*}\left(B_{n} ; \mathbb{Q}\right)$, although we do not use this fact very much in this paper.

We need the following lemma whose proof can be found in [5], Corollary 3.1.

Lemma 4.3. Let $\omega$ be a primitive element of $H^{2}\left(B_{n}\left(\alpha_{1}, \alpha_{2}, \ldots, \alpha_{n}\right) ; \mathbb{Z}\right)$ whose square is zero. Then $\omega$ is either one of the following forms: $\pm\left(x_{i}-\frac{1}{2} \alpha_{i}\right)$, if $\alpha_{i} \equiv 0 \bmod 2$, and $\pm\left(2 x_{i}-\alpha_{i}\right)$, otherwise.

The following lemma from [6], Proposition 4.1 also plays an important role in the proof of Theorem 4.5 .

Lemma 4.4. Let

$$
\psi: H^{*}\left(B_{n}\left(\alpha_{1}, \alpha_{2}, \ldots, \alpha_{n}\right) ; \mathbb{Z}\right) \rightarrow H^{*}\left(B_{n}\left(\alpha_{1}^{\prime}, \alpha_{2}^{\prime}, \ldots, \alpha_{n}^{\prime}\right) ; \mathbb{Z}\right)
$$

be a graded ring isomorphism over integers. Then, for each $1 \leq i \leq n$ there is $r_{i} \in \mathbb{Q}$ such that $\psi\left(y_{i}\right)=r_{i} y_{\sigma(i)}^{\prime}$, where $\sigma$ is a permutation in $S_{n}$.

Proof. For the sake of reader's convenience, we will provide a proof of the lemma, essentially due to that of [6], Proposition 4.1.

For the proof, we shall use the induction on $i$ with $1 \leq i \leq n$. For $i=1$, note that

$$
\psi\left(y_{1}\right)^{2}=\psi\left(y_{1}^{2}\right)=\psi(0)=0
$$

where we used the identities $y_{i}^{2}=\frac{1}{4} \alpha_{i} y_{i}$ and $\alpha_{1}=0$. Then it follows from Lemma 4.3 that there is some $r_{1}$ in $\mathbb{Q}$ such that

$$
\psi\left(y_{1}\right)=r_{1} y_{\sigma(1)}^{\prime}
$$

where $\sigma$ is an element of the symmetric group $S_{n}$. So, we are done with the case of $i=1$.

Next, suppose that the lemma holds for any $i<k$, i.e.,

$$
\psi\left(y_{i}\right)=r_{i} y_{\sigma(i)}^{\prime}, \quad r_{i}, \in \mathbb{Q}, 1 \leq i<k,
$$


where $\sigma$ is an element of the symmetric group $S_{n}$. We then may assume without loss of generality that $\sigma(i)=i$ for any $1 \leq i<k$. It is also easy to obtain

$$
\begin{aligned}
\psi\left(y_{k}\right)^{2} & =\psi\left(y_{k}^{2}\right)=\psi\left(\frac{1}{4} \alpha_{k}^{2}\right) \stackrel{(1.1)}{=} \psi\left(\frac{1}{4}\left(\sum_{i=1}^{k-1} c_{k}^{i} x_{i}\right)^{2}\right) \\
& \stackrel{\left(\text { Lem. }^{3.2)}\right.}{=} \frac{1}{4}\left(\psi\left(\sum_{i=1}^{k-1} \sum_{l=1}^{i} c_{k}^{i} b_{i}^{l} y_{l}\right)\right)^{2}=\frac{1}{4}\left(\psi\left(\sum_{l=1}^{k-1}\left(\sum_{i=l}^{k-1} c_{k}^{i} b_{i}^{l}\right) y_{l}\right)\right)^{2} \\
& =\frac{1}{4}\left(\psi\left(\sum_{l=1}^{k-1} d_{l} y_{l}\right)\right)^{2}=\frac{1}{4} \sum_{t, s=1}^{k-1} d_{t} d_{s} \psi\left(y_{t}\right) \psi\left(y_{s}\right) \\
& =\frac{1}{4} \sum_{t, s=1}^{k-1}\left(d_{t} r_{t}\right)\left(d_{s} r_{s}\right) y_{t}^{\prime} y_{s}^{\prime}, \quad \psi\left(y_{t}\right)=r_{t} y_{t}^{\prime}, \psi\left(y_{s}\right)=r_{s} y_{s}^{\prime}
\end{aligned}
$$

where $d_{l}=\sum_{l=l}^{k-1} c_{k}^{i} b_{i}^{l}$.

Now, let

$$
\psi\left(y_{k}\right)=\sum_{m=1}^{n} e_{m} y_{m}^{\prime}, \quad e_{m} \in \mathbb{Q} .
$$

It is not difficult to see from (4.4) that $e_{m}=0$ at least for all $m \geq k+1$. Thus we have

$$
\psi\left(y_{k}\right)=\sum_{m=1}^{k} e_{m} y_{m}^{\prime} .
$$

We then claim that all of $e_{m}$ are zero for $1 \leq m \leq k-1$. To see it, suppose that there is some non-zero coefficient $e_{m}$ for $1 \leq m \leq k-1$, then there should be a term of the form $A y_{m}^{\prime} y_{k}^{\prime}(A \in \mathbb{Q})$ in the expression of $\psi\left(y_{k}\right)^{2}$. But this contradicts the equation (4.4). Therefore, we have $\psi\left(y_{k}\right)=e_{k} y_{k}^{\prime}$, which implies that, in general, for a permutation $\sigma \in S_{n}$ we have

$$
\psi\left(y_{k}\right)=e_{k} y_{\sigma(k)}^{\prime}, \quad 1 \leq k \leq n .
$$

This completes the proof of Lemma 4.4.

Note that $r_{i}$ in Lemma 4.4 is either $\pm \frac{1}{2}$, or \pm 2 , or \pm 1 (see, e.g., [6], Lemma 4.1). With this understood, we give a partial result, related to Corollary 1.6, for two diffeomorphic Bott manifolds, as follows.

Theorem 4.5. Let $B_{n}\left(\alpha_{1}, \ldots, \alpha_{n}\right)$ be diffeomorphic to $B_{n}\left(\alpha_{1}^{\prime}, \ldots, \alpha_{n}^{\prime}\right)$ by a diffeomorphism $F$, and let

$$
\psi: H^{*}\left(B_{n}\left(\alpha_{1}, \alpha_{2}, \ldots, \alpha_{n}\right) ; \mathbb{Z}\right) \rightarrow H^{*}\left(B_{n}\left(\alpha_{1}^{\prime}, \alpha_{2}^{\prime}, \ldots, \alpha_{n}^{\prime}\right) ; \mathbb{Z}\right)
$$

be the graded ring isomorphism over integers given by the pullback of $F^{-1}$. If all of $r_{i}(1 \leq i \leq n)$ are equal to \pm 1 , then there is some permutation $\sigma \in S_{n}$ 
such that we have

for all $1 \leq i \leq n$.

$$
\psi\left(\alpha_{i}\right) \equiv \alpha_{\sigma(i)}^{\prime} \quad \bmod 2
$$

Proof. To prove it, recall first that $\alpha_{1}=\alpha_{1}^{\prime}=0$ by definition. So the theorem trivially holds for $n=1$.

For the case of $n=2$, by assumption two Bott manifolds $B_{2}\left(\alpha_{1}, \alpha_{2}\right)$ and $B_{2}\left(\alpha_{1}^{\prime}, \alpha_{2}^{\prime}\right)$ are diffeomorphic. Thus there is a graded ring isomorphism $\psi$ from $H^{*}\left(B_{2}\left(\alpha_{1}, \alpha_{2}\right) ; \mathbb{Z}\right)$ and $H^{*}\left(B_{2}\left(\alpha_{1}^{\prime}, \alpha_{2}^{\prime}\right) ; \mathbb{Z}\right)$. It then follows from Lemma 4.4 that there is a permutation $\sigma \in S_{2}$ such that

$$
\psi\left(y_{1}\right)= \pm y_{\sigma(1)}^{\prime}, \text { and } \psi\left(y_{2}\right)= \pm y_{\sigma(2)}^{\prime} .
$$

In other words, we have

$$
\psi\left(2 x_{1}-\alpha_{1}\right)= \pm\left(2 x_{\sigma(1)}^{\prime}-\alpha_{\sigma(1)}^{\prime}\right) \text {, and } \psi\left(2 x_{2}-\alpha_{2}\right)= \pm\left(2 x_{\sigma(2)}^{\prime}-\alpha_{\sigma(2)}^{\prime}\right) .
$$

This implies that

$$
\psi\left(\alpha_{1}\right) \equiv \alpha_{\sigma(1)}^{\prime}, \text { and } \psi\left(\alpha_{2}\right) \equiv \alpha_{\sigma(2)}^{\prime} \bmod 2,
$$

as claimed. It should be now clear that exactly same arguments apply to any general $n \geq 3$. So we are done.

Acknowledgements. The author is very grateful for the anonymous referee for the valuable comments on this paper. This research was supported by Basic Science Research Program through the National Research Foundation of Korea (NRF) funded by the Ministry of Education (2014R1A1A2054683, ASARC, NRF-2007-0056093).

\section{References}

[1] A. Borel and F. Hirzebruch, Characteristic classes and homogeneous spaces. I, Amer. J. Math. 80 (1958), 458-538.

[2] R. Bott and H. Samelson, Applications of the theory of Morse to symmetric spaces, Amer. J. Math. 80 (1958), 964-1029.

[3] R. Bott and L. W. Tu, Differential Forms in Algebraic Topology, Grad. Texts Math. 82, Springer, 1982.

[4] V. Buchstaber and T. Panov, Torus actions and their applications in topology and combinatorics, University Lecture Series, Vol. 24, Amer. Math. Soc., Providence, Rhode Island, 2002.

[5] S. Choi and M. Masuda, Classification of $\mathbb{Q}$-trivial Bott towers, J. Symp. Geom. 10 (2012), no. 3, 447-462.

[6] S. Choi, M. Masuda, and S. Murai, Invariance of Pontrjagin classes for Bott manifolds, Algebr. Geom. Topol. 15 (2015), 965-986.

[7] Y. Civan and N. Ray, Homotopy decompositions and K-theory of Bott towers, $K$-theory 34 (2005), no. 1, 1-33.

[8] M. Davis and T. Januszkiewicz, Convex polytopes, Coxeter orbifolds, and torus actions, Duke Math. J. 62 (1991), no. 2, 417-451.

[9] M. Grossberg and Y. Karshon, Bott towers, complete integrability, and the extended character of representations, Duke Math. J. 76 (1994), no. 1, 23-58. 
[10] F. Hirzebruch, Über eine Klasse von einfachzusammenhängenden komplexen Mannigfaltigkeiten, Math. Ann. 124 (1951), 77-86.

[11] H. Ishida, Filtered cohomological rigidity of Bott towers, Osaka J. Math. 49 (2012), no. $2,515-522$.

[12] J.-H. Kim, On a generalized Petrie's conjecture via index theorems, Topology Appl. 160 (2013), no. 12, 1353-1363.

[13] S. Kuroki and D. Y. Suh, Cohomological non-rigidity of eight-dimensional complex projective towers, Algebr. Geom. Topol. 15 (2015), no. 2, 769-782

[14] M. Masuda and T. Panov, Semi-free circle actions, Bott towers, and quasitoric manifolds, Sb. Math. 199 (2008), no. 7-8, 1201-1223.

[15] M. Masuda and D. Y. Suh, Classification problems of toric manifolds via topology, Toric topology, 273-286, Contemp. Math., 460, Amer. Math. Soc., Providence, RI, 2008.

[16] J. Morrow and K. Kodaira, Complex manifolds, AMS Chelsea Publishing, Amer. Math. Soc., Providence, Rhode Island, 1971.

[17] T. Petrie, Smooth $S^{1}$ actions on homotopy complex projective spaces and related topics, Bull. Amer. Math. Soc. 78 (1972), 105-153.

[18] , Torus actions on homotopy complex projective spaces, Invent. Math. 20 (1973), $139-146$.

[19] F. W. Warner, Foundations of differentiable manifolds and Lie groups, Scott, Foresman and Company, Illinois, 1971.

Department of Mathematics Education

Chosun University

GwanguU 61452, Korea

E-mail address: jinhkim11@gmail.com 